\title{
The Schwarz Type Inequality for Harmonic Mappings of the Unit Disc with Boundary Normalization
}

\author{
Dariusz Partyka • Józef Zając
}

Received: 24 April 2014 / Accepted: 19 June 2014 / Published online: 16 July 2014

(C) The Author(s) 2014. This article is published with open access at Springerlink.com

\begin{abstract}
Let $\mathcal{H}$ be the class of all complex-valued harmonic functions $F$ of the unit disk $\mathbb{D}$ into itself such that for every $k \in\{0,1,2\}$ and almost every $z \in T_{k}:=\left\{\mathrm{e}^{\mathrm{i} \theta}\right.$ : $2 k \pi / 3 \leq \theta \leq 2(k+1) \pi / 3\}$ the radial limit of $F$ at $z$ belongs to the angular sector determined by the convex hull spanned by the origin and arc $T_{k}$. The sharp estimation of the modulus $|F(z)|$ for $z \in \mathbb{D}$ in the class $\mathcal{H}$ is obtained and the extremal functions are determined.
\end{abstract}

Keywords Harmonic mappings · Poisson integral $\cdot$ Schwarz Lemma

Mathematics Subject Classification (2010) Primary 30C55 · 30C62

\section{Introduction}

Let $\mathbb{D}(a, r):=\{z \in \mathbb{C}:|z-a|<r\}$ and $\mathbb{T}(a, r):=\{z \in \mathbb{C}:|z-a|=r\}$ for $a \in \mathbb{C}$ and $r>0$. In particular $\mathbb{D}:=\mathbb{D}(0,1)$ and $\mathbb{T}:=\mathbb{T}(0,1)$ are the unit disk and unit circle, respectively. We denote by $\operatorname{Har}(\mathbb{D})$ the class of all complex-valued harmonic functions in $\mathbb{D}$, i.e., the class of twice continuously differentiable functions $F$ in $\mathbb{D}$

Communicated by David Shoikhet.

D. Partyka · J. Zając $(\varangle)$

Institute of Mathematics and Information Technology, The State School of Higher Education in Chełm, Pocztowa 54, 22-100 Chełm, Poland

e-mail: jzajac@kul.pl

D. Partyka

Institute of Mathematics and Computer Science, The John Paul II Catholic University of Lublin,

Al. Racławickie 14, P.O. Box 129, 20-950 Lublin, Poland

e-mail: partyka@kul.lublin.pl

Birkhäuser 
satisfying the Laplace equation

$$
\frac{\partial^{2}}{\partial x^{2}} F(z)+\frac{\partial^{2}}{\partial y^{2}} F(z)=0, \quad z=x+\mathrm{i} y \in \mathbb{D} .
$$

In 1959 E. Heinz proved in [5] that for every $F \in \operatorname{Har}(\mathbb{D})$, if $F(\mathbb{D}) \subset \mathbb{D}$ and $F(0)=0$, then

$$
|F(z)| \leq \frac{4}{\pi} \arctan |z|, \quad z \in \mathbb{D}
$$

cf. also [4, p. 77] and [8]. Moreover, the above estimate is sharp. The equality in (1.1) holds if

$$
F(\zeta)=\operatorname{Re}\left[\frac{2 \mathrm{i}}{\pi} \log \frac{1+\zeta}{1-\zeta}\right], \quad \zeta \in \mathbb{D},
$$

and if $z \in \mathbb{D} \cap \mathbb{R}$. This result can be treated as a counterpart of the well known Schwarz lemma where holomorphic mappings are replaced by the harmonic ones. The estimation (1.1) can be essentially improved under the additional assumption that $F$ is a quasiconformal mapping of $\mathbb{D}$ onto itself; cf. [8, Thm. 3.3] where the bound of $|F(z)|$ depends on the maximal dilatation $K$ of $F$.

In this paper we intend to find a sort of the estimation (1.1) for $F \in \operatorname{Har}(\mathbb{D})$ where the normalization condition $F(0)=0$ is replaced by a certain boundary one described in Corollary 2.2. Its proof appeals to Theorem 2.1 which gives a Schwarz type estimation for $F$ determined by the Poisson integral of a certain function $f: \mathbb{T} \rightarrow \operatorname{cl}(\mathbb{D})$. Here and subsequently, the symbol $\operatorname{cl}(A)$ stands for the closure of a set $A \subset \mathbb{C}$ in the Euclidian topology. From Corollary 2.2 we infer Corollary 2.4, which deals with harmonic injective mappings of $\mathbb{D}$ onto itself. In particular, Corollary 2.4 leads to the following result: If $F$ is a continuous function in the closed disk $\mathrm{cl}(\mathbb{D})$ onto itself, harmonic and injective in $\mathbb{D}$, and normalized by

$$
F\left(e_{k}\right)=e_{k}:=\mathrm{e}^{2 \pi \mathrm{i} k / 3}, \quad k \in\{0,1,2\},
$$

then

$$
|F(z)| \leq \frac{2}{\pi} \arctan \left(\sqrt{3} \frac{1+|z|}{1-|z|}\right), \quad z \in \mathbb{D} .
$$

This is a direct consequence of Corollary 2.4. The right hand side of the inequality in (1.4) can be replaced by the right hand side of the equality in (2.35) or (2.36); cf. Remark 2.5. The estimations (2.5) and (2.29) are sharp. The extremal functions are described in the last section; cf. Theorem 3.3 and Corollary 3.4.

\section{The Schwarz Type Inequalities}

Given an integrable function $f: \mathbb{T} \rightarrow \mathbb{C}$ we denote by $\mathrm{P}[f](z)$ the Poisson integral of $f$ at $z \in \mathbb{D}$, i.e.,

$$
\mathrm{P}[f](z):=\frac{1}{2 \pi} \int_{\mathbb{T}} f(u) \operatorname{Re} \frac{u+z}{u-z}|\mathrm{~d} u|, \quad z \in \mathbb{D} .
$$


Here and in the sequel integrable means integrable in the sense of Lebesgue. The Poisson integral $\mathrm{P}[f]$ is the unique solution to the Dirichlet problem for the unit disk $\mathbb{D}$ provided the boundary function $f$ is continuous; cf. e.g. [7, Thm. 2.11]. This means that $\mathbf{P}[f]$ is a harmonic mapping in $\mathbb{D}$ which has a continuous extension to the closed disk $\operatorname{cl}(\mathbb{D})$ and its boundary limiting valued function is identical with $f$. Since

$$
\begin{aligned}
\operatorname{Re} \frac{u+z}{u-z} & =\operatorname{Re} \frac{(u+z) \overline{(u-z)}}{|u-z|^{2}}=\frac{|u|^{2}-|z|^{2}}{|u|^{2}-2 \operatorname{Re}(u \bar{z})+|z|^{2}} \\
& =\frac{1-|z|^{2}}{1-2 \operatorname{Re}(z \bar{u})+|z|^{2}}, \quad u \in \mathbb{T}, z \in \mathbb{D},
\end{aligned}
$$

we conclude from (2.1) that

$$
\mathrm{P}[f]\left(r \mathrm{e}^{\mathrm{i} \theta}\right)=\int_{0}^{2 \pi} \mathrm{P}_{r}(\theta-t) f\left(\mathrm{e}^{\mathrm{i} t}\right) \mathrm{d} t, \quad r \mathrm{e}^{\mathrm{i} \theta} \in \mathbb{D},
$$

where

$$
\mathrm{P}_{r}(t):=\frac{1}{2 \pi} \frac{1-r^{2}}{1-2 r \cos t+r^{2}}, \quad r \mathrm{e}^{\mathrm{i} t} \in \mathbb{D},
$$

Setting $I_{k}:=[2 \pi k / 3 ; 2 \pi(k+1) / 3]$ for $k \in\{0,1,2\}$ we define

$$
T_{k}:=\left\{\mathrm{e}^{\mathrm{i} t}: t \in I_{k}\right\} \quad \text { and } \quad D_{k}:=\left\{r \mathrm{e}^{\mathrm{i} t}: t \in I_{k}, r \in[0 ; 1]\right\}, \quad k \in\{0,1,2\} .
$$

Theorem 2.1 For any integrable function $f: \mathbb{T} \rightarrow \mathbb{C}$, if

$$
f(z) \in D_{k} \text { for a.e. } z \in T_{k}, \quad k \in\{1,2,3\}
$$

then

$$
|\mathrm{P}[f](z)| \leq \frac{4}{3}-\frac{2}{\pi} \arctan \left(\frac{\sqrt{3}}{1+2|z|}\right), \quad z \in \mathbb{D},
$$

and the estimation is sharp.

Proof Given a function $f: \mathbb{T} \rightarrow \mathbb{C}$ satisfying the condition (2.4) we consider the function $F:=\mathrm{P}[f]$. Fix $r \in[0 ; 1)$. Since the function $\mathbb{R} \ni \theta \mapsto\left|F\left(r \mathrm{e}^{\mathrm{i} \theta}\right)\right|$ is continuous and $2 \pi$-periodical we have

$$
\left|F\left(r \mathrm{e}^{\mathrm{i} \theta}\right)\right| \leq\left|F\left(r \mathrm{e}^{\mathrm{i} \theta_{r}}\right)\right|, \quad \theta \in \mathbb{R}
$$

for a certain $\theta_{r} \in[0 ; 2 \pi)$. On the other hand, there exists $\alpha \in[0 ; 2 \pi)$ such that

$$
F\left(r \mathrm{e}^{\mathrm{i} \theta_{r}}\right)=\mathrm{e}^{\mathrm{i} \alpha}\left|F\left(r \mathrm{e}^{\mathrm{i} \theta_{r}}\right)\right| .
$$

Therefore

$$
\left|F\left(r \mathrm{e}^{\mathrm{i} \theta_{r}}\right)\right|=\operatorname{Re}\left(\mathrm{e}^{-\mathrm{i} \alpha} F\left(r \mathrm{e}^{\mathrm{i} \theta_{r}}\right)\right) .
$$


Hence and by (2.6),

$$
\left|F\left(r \mathrm{e}^{\mathrm{i} \theta}\right)\right| \leq \operatorname{Re}\left(\mathrm{e}^{-\mathrm{i} \alpha} F\left(r \mathrm{e}^{\mathrm{i} \theta_{r}}\right)\right)=\operatorname{Re}\left(\mathrm{e}^{-\mathrm{i} \alpha} \mathrm{P}[f]\left(r \mathrm{e}^{\mathrm{i} \theta_{r}}\right)\right) .
$$

Setting

$$
p_{k}:=\int_{I_{k}} \mathrm{P}_{r}\left(\theta_{r}-t\right) \mathrm{d} t, \quad k \in\{0,1,2\},
$$

we conclude from the formula (2.2) that

$$
\begin{aligned}
\mathrm{P}[f]\left(r \mathrm{e}^{\mathrm{i} \theta_{r}}\right) & =\int_{0}^{2 \pi} \mathrm{P}_{r}\left(\theta_{r}-t\right) f\left(\mathrm{e}^{\mathrm{i} t}\right) \mathrm{d} t \\
& =\sum_{k=0}^{2} p_{k} \int_{I_{k}} \frac{1}{p_{k}} \mathrm{P}_{r}\left(\theta_{r}-t\right) f\left(\mathrm{e}^{\mathrm{i} t}\right) \mathrm{d} t .
\end{aligned}
$$

Since each sector $D_{k}, k \in\{0,1,2\}$, is closed and convex in $\mathbb{C}$ we conclude from the assumption (2.4) and the integral mean value theorem for complex-valued functions that there exist $\alpha_{k} \in I_{k}$ and $r_{k} \in[0 ; 1]$ for $k \in\{0,1,2\}$ such that

$$
r_{k} \mathrm{e}^{\mathrm{i} \alpha_{k}}=\int_{I_{k}} \frac{1}{p_{k}} \mathrm{P}_{r}\left(\theta_{r}-t\right) f\left(\mathrm{e}^{\mathrm{i} t}\right) \mathrm{d} t, \quad k \in\{0,1,2\} .
$$

Combining this with (2.8) and (2.10) we obtain

$$
\left|F\left(r \mathrm{e}^{\mathrm{i} \theta}\right)\right| \leq \operatorname{Re}\left[\mathrm{e}^{-\mathrm{i} \alpha} \sum_{k=0}^{2} p_{k} r_{k} \mathrm{e}^{\mathrm{i} \alpha_{k}}\right]=\sum_{k=0}^{2} c_{k} p_{k}, \quad \theta \in \mathbb{R}
$$

where

$$
\left.c_{k}:=\operatorname{Re}\left(\mathrm{e}^{-\mathrm{i} \alpha} r_{k} \mathrm{e}^{\mathrm{i} \alpha_{k}}\right)\right)=r_{k} \operatorname{Re}\left(\mathrm{e}^{\mathrm{i}\left(\alpha_{k}-\alpha\right)}\right)=r_{k} \cos \left(\alpha_{k}-\alpha\right), \quad k \in\{0,1,2\} .
$$

If $\min \left(\left\{c_{0}, c_{1}, c_{2}\right)\right\} \leq 0$, then clearly

$$
c_{0}+c_{1}+c_{2} \leq 2
$$

Therefore, we can assume that all $c_{0}, c_{1}, c_{2}$ are positive, and consequently

$$
c_{k} \leq \cos \left(\alpha_{k}-\alpha\right), \quad k \in\{0,1,2\}
$$

because $r_{k} \in[0 ; 1]$ for $k \in\{0,1,2\}$. Since $\alpha_{k} \in I_{k}$ for $k \in\{0,1,2\}$, we see that

$$
\frac{2 \pi k}{3}-\alpha \leq \alpha_{k}-\alpha \leq \frac{2 \pi(k+1)}{3}-\alpha, \quad k \in\{0,1,2\} .
$$


If $0 \leq \alpha \leq 2 \pi / 3$, then by (2.15) and (2.16),

$$
c_{0} \leq 1, \quad c_{1} \leq \cos \left(\frac{2 \pi}{3}-\alpha\right) \text { and } \quad c_{2} \leq \cos (2 \pi-\alpha)=\cos (\alpha)
$$

and so

$$
c_{0}+c_{1}+c_{2} \leq 1+\cos \left(\frac{2 \pi}{3}-\alpha\right)+\cos (\alpha)=1+2 \cos \frac{\pi}{3} \cos \left(\frac{\pi}{3}-\alpha\right) \leq 2
$$

If $2 \pi / 3 \leq \alpha \leq 4 \pi / 3$, then by (2.15) and (2.16),

$$
c_{0} \leq \cos \left(\frac{2 \pi}{3}-\alpha\right), \quad c_{1} \leq 1 \quad \text { and } \quad c_{2} \leq \cos \left(\frac{4 \pi}{3}-\alpha\right)
$$

and so

$c_{0}+c_{1}+c_{2} \leq 1+\cos \left(\frac{2 \pi}{3}-\alpha\right)+\cos \left(\frac{4 \pi}{3}-\alpha\right)=1+2 \cos \frac{\pi}{3} \cos (\pi-\alpha) \leq 2$.

If $4 \pi / 3 \leq \alpha \leq 2 \pi$, then by (2.15) and (2.16),

$$
c_{0} \leq \cos (\alpha), \quad c_{1} \leq \cos \left(\frac{4 \pi}{3}-\alpha\right) \quad \text { and } \quad c_{2} \leq 1
$$

and so

$$
c_{0}+c_{1}+c_{2} \leq 1+\cos (\alpha)+\cos \left(\frac{4 \pi}{3}-\alpha\right)=1+2 \cos \frac{2 \pi}{3} \cos \left(\frac{2 \pi}{3}-\alpha\right) \leq 2 .
$$

Thus in all the cases the inequality (2.14) holds. In order to estimate the last sum in (2.12) we consider the function

$$
\mathbb{R} \ni \theta \mapsto Q_{r}(\theta):=\int_{2 \pi / 3}^{4 \pi / 3} \mathrm{P}_{r}(\theta-t) \mathrm{d} t=\mathrm{P}\left[\chi_{T_{1}}\right]\left(r \mathrm{e}^{\mathrm{i} \theta}\right)
$$

where $\chi_{A}$ is the characteristic function of a set $A \subset \mathbb{T}$, i.e., $\chi_{A}(t):=1$ for $t \in A$ and $\chi_{A}(t):=0$ for $t \in \mathbb{T} \backslash A$. Throughout the paper we understand the function log as the inverse function to $\exp _{\mid \Omega}$, where $\Omega:=\{z \in \mathbb{C}:|\operatorname{Im} z|<\pi\}$. Given $z \in \mathbb{D}$ we see that $T_{1} \subset \Omega_{z}:=\mathbb{C} \backslash\{z+t: t>0\}$, the function $\Omega_{z} \ni \zeta \mapsto \log (z-\zeta)$ is holomorphic and

$$
\frac{\mathrm{d}}{\mathrm{d} t} \log \left(z-\mathrm{e}^{\mathrm{i} t}\right)=\frac{\mathrm{i} \mathrm{e}^{\mathrm{i} t}}{\mathrm{e}^{\mathrm{i} t}-z}, \quad t \in I_{1} .
$$


Hence and by the formula (2.1) we see that

$$
\begin{aligned}
\mathrm{P}\left[\chi_{T_{1}}\right](z) & =\frac{1}{2 \pi} \int_{T_{1}} \chi_{T_{1}}(u) \operatorname{Re} \frac{u+z}{u-z}|\mathrm{~d} u| \\
& =\frac{1}{2 \pi} \int_{2 \pi / 3}^{4 \pi / 3} \operatorname{Re} \frac{\mathrm{e}^{\mathrm{i} t}+z}{\mathrm{e}^{\mathrm{i} t}-z} \mathrm{~d} t \\
& =\frac{1}{2 \pi} \int_{2 \pi / 3}^{4 \pi / 3} \operatorname{Re}\left(\frac{2 \mathrm{e}^{\mathrm{i} t}}{\mathrm{e}^{\mathrm{i} t}-z}-1\right) \mathrm{d} t \\
& =\frac{1}{\pi} \int_{2 \pi / 3}^{4 \pi / 3} \operatorname{Im}\left(\frac{\mathrm{i} \mathrm{e}^{\mathrm{i} t}}{\mathrm{e}^{\mathrm{i} t}-z}\right) \mathrm{d} t-\frac{1}{3} \\
& =\frac{1}{\pi} \int_{2 \pi / 3}^{4 \pi / 3} \operatorname{Im} \frac{\mathrm{d}}{\mathrm{d} t} \log \left(z-\mathrm{e}^{\mathrm{i} t}\right) \mathrm{d} t-\frac{1}{3} \\
& =\frac{1}{\pi} \operatorname{Im}\left[\log \left(z-e_{2}\right)-\log \left(z-e_{1}\right)\right]-\frac{1}{3} .
\end{aligned}
$$

Hence and by (2.17),

$$
Q_{r}(\theta)=\frac{1}{\pi} \operatorname{Im}\left[\log \left(r \mathrm{e}^{\mathrm{i} \theta}-e_{2}\right)-\log \left(r \mathrm{e}^{\mathrm{i} \theta}-e_{1}\right)\right]-\frac{1}{3}, \quad \theta \in \mathbb{R},
$$

and consequently,

$$
\begin{aligned}
\frac{\mathrm{d}}{\mathrm{d} \theta} Q_{r}(\theta) & =\frac{1}{\pi} \operatorname{Im}\left[\frac{\mathrm{i} r \mathrm{e}^{\mathrm{i} \theta}}{r \mathrm{e}^{\mathrm{i} \theta}-e_{2}}-\frac{\mathrm{i} r \mathrm{e}^{\mathrm{i} \theta}}{r \mathrm{e}^{\mathrm{i} \theta}-e_{1}}\right] \\
& =\frac{r}{\pi} \operatorname{Im}\left[\frac{\mathrm{ie}^{\mathrm{i} \theta}\left(e_{2}-e_{1}\right)}{\left(r \mathrm{e}^{\mathrm{i} \theta}-e_{2}\right)\left(r \mathrm{e}^{\mathrm{i} \theta}-e_{1}\right)}\right] \\
& =\frac{r \sqrt{3}}{\pi} \frac{\operatorname{Im}\left[\mathrm{e}^{\mathrm{i} \theta}\left(r \mathrm{e}^{-\mathrm{i} \theta}-e_{1}\right)\left(r \mathrm{e}^{-\mathrm{i} \theta}-e_{2}\right)\right.}{\left|r \mathrm{e}^{\mathrm{i} \theta}-e_{2}\right|^{2}\left|r \mathrm{e}^{\mathrm{i} \theta}-e_{1}\right|^{2}} \\
& =\frac{r \sqrt{3}}{\pi} \frac{\operatorname{Im}\left[r^{2} \mathrm{e}^{-\mathrm{i} \theta}+r+\mathrm{e}^{\mathrm{i} \theta}\right]}{\left|r \mathrm{e}^{\mathrm{i} \theta}-e_{2}\right|^{2}\left|r \mathrm{e}^{\mathrm{i} \theta}-e_{1}\right|^{2}} \\
& =\frac{r \sqrt{3}\left(1-r^{2}\right) \sin \theta}{\pi\left|r \mathrm{e}^{\mathrm{i} \theta}-e_{2}\right|^{2}\left|r \mathrm{e}^{\mathrm{i} \theta}-e_{1}\right|^{2}}, \quad \theta \in \mathbb{R} .
\end{aligned}
$$


Combining this with (2.18) we obtain

$$
\begin{aligned}
Q_{r}(\theta) \geq Q_{r}(0) & =\frac{1}{\pi} \operatorname{Im}\left[\log \left(r-e_{2}\right)-\log \left(r-e_{1}\right)\right]-\frac{1}{3} \\
& =\frac{2}{\pi} \arctan \frac{\sqrt{3}}{1+2 r}-\frac{1}{3}, \quad \theta \in \mathbb{R} .
\end{aligned}
$$

Combining (2.9) with (2.17) we have

$$
\begin{aligned}
& p_{0}=\int_{I_{0}} \mathrm{P}_{r}\left(\theta_{r}-t\right) \mathrm{d} t=\int_{I_{1}} \mathrm{P}_{r}\left(\theta_{r}+2 \pi / 3-t\right) \mathrm{d} t=Q_{r}\left(\theta_{r}+2 \pi / 3\right), \\
& p_{1}=\int_{I_{1}} \mathrm{P}_{r}\left(\theta_{r}-t\right) \mathrm{d} t=Q_{r}\left(\theta_{r}\right), \\
& p_{2}=\int_{I_{2}} \mathrm{P}_{r}\left(\theta_{r}-t\right) \mathrm{d} t=\int_{I_{1}} \mathrm{P}_{r}\left(\theta_{r}-2 \pi / 3-t\right) \mathrm{d} t=Q_{r}\left(\theta_{r}-2 \pi / 3\right) .
\end{aligned}
$$

Hence and by (2.20),

$$
\min \left(\left\{p_{0}, p_{1}, p_{2}\right\}\right) \geq Q_{r}(0)=\frac{2}{\pi} \arctan \frac{\sqrt{3}}{1+2 r}-\frac{1}{3} .
$$

From (2.9) it follows that

$$
p_{0}+p_{1}+p_{2}=\sum_{k=0}^{2} \int_{I_{k}} \mathrm{P}_{r}\left(\theta_{r}-t\right) \mathrm{d} t=\int_{0}^{2 \pi} \mathrm{P}_{r}\left(\theta_{r}-t\right) \mathrm{d} t=1 .
$$

This together with (2.22), (2.14) and (2.15) leads to

$$
\begin{aligned}
\sum_{k=0}^{2} c_{k} p_{k} & =\sum_{k=0}^{2}\left(p_{k}-Q_{r}(0)\right) c_{k}+\sum_{k=0}^{2} Q_{r}(0) c_{k} \\
& \leq \sum_{k=0}^{2}\left(p_{k}-Q_{r}(0)\right)+Q_{r}(0) \sum_{k=0}^{2} c_{k} \\
& \leq \sum_{k=0}^{2} p_{k}-\sum_{k=0}^{2} Q_{r}(0)+2 Q_{r}(0) \\
& =1-Q_{r}(0) .
\end{aligned}
$$

Combining (2.12) with (2.24) and (2.20) we infer the estimation (2.5). It is clear that the function

$$
f_{0}:=\chi_{T_{0} \cup T_{2}}
$$


satisfies the condition (2.4) with $f:=f_{0}$. From the formula (2.17) it follows that for every $z=r \mathrm{e}^{\mathrm{i} \theta} \in \mathbb{D}$,

$$
\mathrm{P}\left[f_{0}\right](z)=\mathrm{P}\left[\chi_{\mathbb{T} \backslash T_{1}}\right](z)=\mathrm{P}\left[\chi_{\mathbb{T}}-\chi_{T_{1}}\right](z)=1-\mathrm{P}\left[\chi_{T_{1}}\right](z)=1-Q_{r}(\theta) .
$$

Hence and by the equalities in (2.20) we conclude that

$$
\mathrm{P}\left[f_{0}\right](r)=1-Q_{r}(0)=\frac{4}{3}-\frac{2}{\pi} \arctan \frac{\sqrt{3}}{1+2 r}, \quad r \in[0 ; 1) .
$$

This means that $f_{0}$ is an extremal function in Theorem 2.1, and so the estimation (2.5) is sharp, which completes the proof.

Now we are ready to prove our main result.

Corollary 2.2 Let $F \in \operatorname{Har}(\mathbb{D})$ satisfy $F(\mathbb{D}) \subset \mathbb{D}$ and for every $k \in\{0,1,2\}$,

$$
\lim _{r \rightarrow 1^{-}} F(r z) \in D_{k} \text { for a.e. } z \in T_{k} \cap \Lambda(F)
$$

where $\Lambda(F)$ is the set of all $z \in \mathbb{T}$ such that the limit exists. Then

$$
|F(z)| \leq \frac{4}{3}-\frac{2}{\pi} \arctan \left(\frac{\sqrt{3}}{1+2|z|}\right), \quad z \in \mathbb{D}
$$

and the estimation is sharp.

Proof Fix a harmonic function $F: \mathbb{D} \rightarrow \mathbb{D}$ satisfying the assumptions of the theorem. Then $\operatorname{Re} F$ and $\operatorname{Im} F$ are real-valued and bounded harmonic functions in $\mathbb{D}$, and so they have a radial limit a.e. in $\mathbb{T}$; cf. [3, Cor. 1 , Sect. 1.2]. Therefore, there exists the limit in (2.28) for a.e. $z \in \mathbb{T}$. Setting

$$
\mathbb{T} \ni z \mapsto f(z):= \begin{cases}\lim _{r \rightarrow 1^{-}} F(r z) & \text { for } z \in \Lambda(F), \\ 0 & \text { for } z \in \mathbb{T} \backslash \Lambda(F),\end{cases}
$$

we see that the function $f$ is measurable and bounded. Since $F(\mathbb{D}) \subset \mathbb{D}$, we can apply the dominated convergence theorem to show that $F=\mathrm{P}[f]$. From the condition (2.28) it follows that $f(z) \in D_{k}$ for a.e. $z \in T_{k}$ and every $k \in\{0,1,2\}$. Theorem 2.1 now implies the estimation (2.29).

From the properties of the Poisson integral it follows that the function

$$
\mathbb{D} \ni z \mapsto F_{0}(z):=\mathrm{P}\left[\chi_{T_{0} \cup T_{2}}\right](z)
$$

is harmonic in $\mathbb{D}, F_{0}(\mathbb{D}) \subset \mathbb{D}$ and

$$
\lim _{r \rightarrow 1^{-}} F_{0}(r z)=\mathrm{P}\left[\chi_{T_{0} \cup T_{2}}\right](z), \quad z \in \mathbb{T} \backslash\left\{e_{1}, e_{2}\right\}
$$


Therefore the function $F_{0}$ satisfies the condition (2.28) with $F$ replaced by $F_{0}$. Combining (2.30) with (2.25) and (2.27) we see that

$$
F_{0}(r)=\frac{4}{3}-\frac{2}{\pi} \arctan \frac{\sqrt{3}}{1+2 r}, \quad r \in[0 ; 1) .
$$

This means that $F_{0}$ is an extremal function in Corollary 2.2, and so the estimation (2.29) is sharp, which completes the proof.

Remark 2.3 Let $\mathcal{H}$ be the class of all $F \in \operatorname{Har}(\mathbb{D})$ satisfying the assumption of Corollary 2.2. Applying (2.29) for $z:=0$ we obtain the following useful estimation

$$
|F(0)| \leq \frac{2}{3}, \quad F \in \mathcal{H}
$$

which is best possible. In other words, the variability region of the origin for the class $\mathcal{H}$ satisfies the inclusion

$$
\{F(0): F \in \mathcal{H}\} \subset \operatorname{cl}(\mathbb{D}(0,2 / 3))
$$

and $2 / 3$ is the smallest radius of the inclusion.

Corollary 2.4 Let $F \in \operatorname{Har}(\mathbb{D})$ be an injective mapping in $\mathbb{D}$ such that $F(\mathbb{D})=\mathbb{D}$ and

$$
\lim _{r \rightarrow 1^{-}} F\left(r e_{k}\right)=e_{k}, \quad k \in\{0,1,2\} .
$$

Then the estimation (2.29) holds.

Proof Given a mapping $F \in \operatorname{Har}(\mathbb{D})$ suppose that $F$ is injective in $\mathbb{D}$ and $F(\mathbb{D})=\mathbb{D}$. Then, as shown by Choquet in [2], there exists a continuous mapping $f: \mathbb{T} \rightarrow \mathbb{T}$ such that $F=\mathrm{P}[f], f(\mathbb{T})=\mathbb{T}$ and for each $w \in \mathbb{T}, f^{-1}(\{w\})$ is a connected subset of $\mathbb{T}$. Therefore, $f^{-1}(\{w\})$ is a closed arc of $\mathbb{T}$ for $w \in \mathbb{T}$. This property can be also deduced from a more general result; cf. [6] or [4, Sect. 3.3]. Moreover, from the boundary normalization condition (2.32) it follows, that

$$
f\left(e_{k}\right)=\lim _{r \rightarrow 1^{-}} \mathrm{P}[f]\left(r e_{k}\right)=\lim _{r \rightarrow 1^{-}} F\left(r e_{k}\right)=e_{k}, \quad k \in\{0,1,2\} .
$$

Suppose that $f^{-1}\left(\left\{e_{2}\right\}\right) \cap T_{0} \neq \varnothing$. Since $f^{-1}\left(\left\{e_{2}\right\}\right)$ is a closed arc of $\mathbb{T}$, we see that $e_{0} \in f^{-1}\left(\left\{e_{2}\right\}\right)$ or $e_{1} \in f^{-1}\left(\left\{e_{2}\right\}\right)$. Hence $f\left(e_{0}\right)=e_{2}$ or $f\left(e_{1}\right)=e_{2}$, which contradicts the equalities (2.33). Thus $e_{2} \notin f\left(T_{0}\right)$. Moreover, by the continuity of $f$ and by (2.33) we see that $f\left(T_{0}\right)$ is a closed arc of $\mathbb{T}$ containing the points $e_{0}$ and $e_{1}$. Consequently,

$$
T_{0} \subset f\left(T_{0}\right) \subset \mathbb{T} \backslash\left\{e_{2}\right\} .
$$

Suppose that $f\left(T_{0}\right) \backslash T_{0} \neq \emptyset$. Since

$$
f\left(T_{0} \backslash f^{-1}\left(\left\{e_{0}, e_{1}\right\}\right)\right)=f\left(T_{0}\right) \backslash\left\{e_{0}, e_{1}\right\},
$$


we conclude from (2.34) that $f\left(T_{0} \backslash f^{-1}\left(\left\{e_{0}, e_{1}\right\}\right)\right)$ is not a connected subset of $\mathbb{T}$. This is impossible, because $T_{0} \backslash f^{-1}\left(\left\{e_{0}, e_{1}\right\}\right)$ is an open arc of $\mathbb{T}$, and so is the set $f\left(T_{0} \backslash f^{-1}\left(\left\{e_{0}, e_{1}\right\}\right)\right)$. Therefore, $f\left(T_{0}\right) \subset T_{0}$. The same conclusion can be drawn for the $\operatorname{arcs} T_{1}$ and $T_{2}$ in place of $T_{0}$, which means that

$$
f\left(T_{k}\right) \subset T_{k}, \quad k \in\{0,1,2\} .
$$

It follows that for every $k \in\{0,1,2\}$,

$$
\lim _{r \rightarrow 1^{-}} F(r z)=f(z) \in D_{k}, \quad z \in T_{k}
$$

and consequently the condition (2.28) holds. Then Corollary 2.2 yields the estimation (2.29), which proves the corollary.

Remark 2.5 Using the trigonometric identity

$$
\tan (\alpha-\beta)=\frac{\tan \alpha-\tan \beta}{1+\tan \alpha \tan \beta}, \quad \alpha, \beta, \alpha-\beta \in \mathbb{R} \backslash\{n \pi+\pi / 2: n \in \mathbb{Z}\},
$$

we can transform the right hand side of the inequality in (2.29) as follows

$$
\frac{4}{3}-\frac{2}{\pi} \arctan \left(\frac{\sqrt{3}}{1+2|z|}\right)=\frac{2}{\pi} \arctan \left(\sqrt{3} \frac{1+|z|}{1-|z|}\right), \quad z \in \mathbb{D} .
$$

Let us recall that the hyperbolic metric $\rho_{h}$ in $\mathbb{D}$ is given by the formula

$$
\rho_{h}(z, w):=\frac{1}{2} \log \frac{|1-z \bar{w}|+|z-w|}{|1-z \bar{w}|-|z-w|}, \quad z, w \in \mathbb{D} ;
$$

cf. e.g. [1]. Hence

$$
\mathrm{e}^{2 \rho_{h}(z, 0)}=\frac{1+|z|}{1-|z|}, \quad z \in \mathbb{D},
$$

which together with (2.35) leads to

$$
\frac{4}{3}-\frac{2}{\pi} \arctan \left(\frac{\sqrt{3}}{1+2|z|}\right)=\frac{2}{\pi} \arctan \left(\sqrt{3} \mathrm{e}^{2 \rho_{h}(z, 0)}\right), \quad z \in \mathbb{D} .
$$

Therefore the right hand side of the inequalities in (2.5) and (2.29) can be replaced by the right hand side of the equality in (2.35) or (2.36).

\section{The Extremal Functions}

As shown in the proof of Theorem 2.1 the function $f_{0}$, given by the formula (2.25), yields the equality in (2.5). Consequently, the function $F_{0}$, given by the formula (2.30), 
is extremal in Corollary 2.2. Since for every $z=r \mathrm{e}^{\mathrm{i} \theta} \in \mathbb{D}$ the equalities (2.26) hold, we conclude from $(2.18)$ that

$$
F_{0}(z)=1-Q_{r}(\theta)=\frac{4}{3}-\frac{1}{\pi} \operatorname{Im}\left[\log \left(z-e_{2}\right)-\log \left(z-e_{1}\right)\right], \quad z=r \mathrm{e}^{\mathrm{i} \theta} \in \mathbb{D} .
$$

We want to find all extremal functions in Theorem 2.1 and Corollary 2.2. To this end we need the following lemma.

Lemma 3.1 Given a closed arc $T \subset \mathbb{T}$ of length less than $\pi, a \in \mathbb{D}, b \in T \cup\{0\}$ and an integrable function $f: \mathbb{T} \rightarrow \operatorname{cl}(\mathbb{D})$ suppose that

$$
f(z) \in D:=\{r \zeta: \zeta \in T, r \in[0 ; 1]\} \text { for a.e. } z \in T
$$

as well as

$$
\mathrm{P}\left[f \cdot \chi_{T}\right](a)=b \mathrm{P}\left[\chi_{T}\right](a) .
$$

Then $f(z)=$ for a.e. $z \in T$.

Proof Let $T, a, b$ and $f$ satisfy the assumptions. Then $T=\left\{\mathrm{e}^{\mathrm{i} t}: t \in\left[\alpha_{0}-\alpha ; \alpha_{0}+\alpha\right]\right\}$ for some $\alpha_{0} \in \mathbb{R}$ and $\alpha \in[0 ; \pi / 2)$, and $D$ is a convex and closed subset of $\operatorname{cl}(\mathbb{D})$. From (3.2) it follows that

$$
\lambda_{b} \circ f(z) \in \lambda_{b}(D) \subset\{0\} \cup\{\zeta \in \mathbb{C}: \operatorname{Re} \zeta>0\} \text { for a.e. } z \in T,
$$

where $\mathbb{C} \ni \zeta \mapsto \lambda_{0}(\zeta):=\mathrm{e}^{-\mathrm{i} \alpha_{0}} \zeta$ and $\mathbb{C} \ni \zeta \mapsto \lambda_{p}(\zeta):=1-\zeta / p$ for $p \in \mathbb{T}$.

From (3.4) it follows that $\operatorname{Re} \lambda_{b}(f(z)) \geq 0$ for a.e. $z \in T$. By the equality (3.3) we have

$$
\frac{\mathrm{P}\left[\operatorname{Re}\left(\left(\lambda_{b} \circ f\right) \cdot \chi_{T}\right)\right](a)}{\mathrm{P}\left[\chi_{T}\right](a)}=\operatorname{Re}\left(\lambda_{b}\left(\frac{\mathrm{P}\left[f \cdot \chi_{T}\right](a)}{\mathrm{P}\left[\chi_{T}\right](a)}\right)\right)=\operatorname{Re} \lambda_{b}(b)=0 .
$$

Hence the real-valued harmonic function $\mathrm{P}\left[\operatorname{Re}\left(\left(\lambda_{b} \circ f\right) \cdot \chi_{T}\right)\right]$ attains minimum at $a$, and so, by the minimum principle for real-valued harmonic functions,

$$
\mathrm{P}\left[\operatorname{Re}\left(\left(\lambda_{b} \circ f\right) \cdot \chi_{T}\right)\right](z)=0, \quad z \in \mathbb{D} .
$$

Then for a.e. $z \in \mathbb{T}$,

$$
\operatorname{Re} \lambda_{b}(f(z))=\operatorname{Re}\left(\left(\lambda_{b} \circ f\right) \cdot \chi_{T}\right)(z)=\lim _{r \rightarrow 1^{-}} \mathrm{P}\left[\operatorname{Re}\left(\left(\lambda_{b} \circ f\right) \cdot \chi_{T}\right)\right](r z)=0 ;
$$

cf. [3, Cor. 2, Sect. 1.2]. This together with (3.4) leads to $\lambda_{0}(f(z))=0$ for a.e. $z \in T$. Hence $f(z)=b$ for a.e. $z \in T$, which is the desired conclusion.

Remark 3.2 Setting

$$
\mathbb{C} \ni z \mapsto S_{k}(z):=e_{k} z, \quad k \in\{0,1,2\},
$$


we see that the set $\left\{S_{0}, S_{1}, S_{2}\right\}$ is closed with respect to the composition operation of mappings o, i.e., $\left(\left\{S_{0}, S_{1}, S_{2}\right\}\right.$, o $)$ is a group. Let $\mathcal{F}$ be the class of all integrable functions $f: \mathbb{T} \rightarrow \mathbb{C}$ satisfying the condition (2.4). It is easily seen that

$$
S_{k} \circ f \circ S_{k}^{-1} \in \mathcal{F}, \quad f \in \mathcal{F}, k \in\{0,1,2\} .
$$

By the definition of the class $\mathcal{H}$ (see Remark 2.3) we also have

$$
S_{k} \circ F \circ S_{k}^{-1} \in \mathcal{H}, \quad F \in \mathcal{H}, k \in\{0,1,2\} .
$$

From the properties of the Poisson integral it may be also concluded that

$$
\mathrm{P}\left[S_{k} \circ f \circ S_{k}^{-1}\right]=S_{k} \circ \mathrm{P}[f] \circ S_{k}^{-1}, \quad f \in \mathcal{F}, k \in\{0,1,2\} .
$$

Theorem 3.3 Let $f: \mathbb{T} \rightarrow \mathbb{C}$ be an integrable function which satisfies the condition (2.4). Then

$$
|\mathrm{P}[f](z)|=\frac{4}{3}-\frac{2}{\pi} \arctan \left(\frac{\sqrt{3}}{1+2|z|}\right), \quad z \in \mathbb{D},
$$

if and only if there exists $k \in\{0,1,2\}$ such that one of the two following conditions holds:

(i) $z \neq 0$ and

$$
z=S_{k}(|z|) \text { and } f(u)=S_{k} \circ f_{0} \circ S_{k}^{-1}(u) \text { for a.e. } u \in \mathbb{T} \text {, }
$$

where $f_{0}$ is the function given by the formula (2.25);

(ii) $z=0$ and

$$
f(u)=S_{k} \circ f_{0} \circ S_{k}^{-1}(u) \quad \text { or } f(u)=S_{k} \circ f_{0}^{*} \circ S_{k}^{-1}(u) \text { for a.e. } u \in \mathbb{T},
$$

where

$$
f_{0}^{*}:=\mathrm{e}^{\pi \mathrm{i} / 3} \cdot \chi_{T_{0}}+e_{1} \cdot \chi_{T_{1}}+e_{0} \cdot \chi_{T_{2}} .
$$

Proof Fix $z \in \mathbb{D}$ and $f \in \mathcal{F}$. As shown in the final part of the proof of Theorem 2.1 the function $f_{0}$ satisfies (2.27). By the formula (3.12) we have

$$
\begin{aligned}
\left|\mathrm{P}\left[f_{0}^{*}\right](0)\right| & =\left|\mathrm{e}^{\pi \mathrm{i} / 3} \mathrm{P}\left[\chi_{T_{0}}\right](0)+e_{1} \mathrm{P}\left[\chi_{T_{1}}\right](0)+e_{0} \mathrm{P}\left[\chi_{T_{1}}\right](0)\right| \\
& =\frac{1}{3}\left|\mathrm{e}^{\pi \mathrm{i} / 3}+e_{1}+e_{0}\right|=\frac{1}{3}\left|1+\mathrm{e}^{\pi \mathrm{i} / 3}+\mathrm{e}^{-\pi \mathrm{i} / 3}\right|=\frac{2}{3}=\left|\mathrm{P}\left[f_{0}\right](0)\right| .
\end{aligned}
$$

Applying now the property (3.8) we deduce the equality (3.9) from the condition (i), provided $z \neq 0$, and from the condition (ii), provided $z=0$.

Conversely, assume that the equality (3.9) holds. Analyzing respective parts in the proof of Theorem 2.1 we conclude from (2.12), (2.24) and the second equality in (2.27) that 


$$
\sum_{k=0}^{2}\left(p_{k}-Q_{r}(0)\right) c_{k}+\sum_{k=0}^{2} Q_{r}(0) c_{k}=\sum_{k=0}^{2}\left(p_{k}-Q_{r}(0)\right)+2 Q_{r}(0)
$$

and consequently

$$
\sum_{k=0}^{2}\left(p_{k}-Q_{r}(0)\right)\left(1-c_{k}\right)+Q_{r}(0)\left(2-\sum_{k=0}^{2} c_{k}\right)=0 .
$$

Combining this with (2.14), (2.15) and (2.22) we see that

$$
c_{0}+c_{1}+c_{2}=2
$$

and

$$
\left(p_{k}-Q_{r}(0)\right)\left(1-c_{k}\right)=0, \quad k \in\{0,1,2\} .
$$

Since $c_{k} \leq 1$ for $k \in\{0,1,2\}$, the equality (3.13) is impossible provided $c:=\min \left(\left\{c_{0}, c_{1}, c_{2}\right\}\right)<0$. Therefore $0 \leq c \leq 1$. Suppose that $c=0$. Then $c_{0}=0$ or $c_{1}=0$ or $c_{2}=0$.

If $c_{0}=0$, then by (3.13), $c_{1}=c_{2}=1$, which leads, by (2.13), to

$$
r_{0}=0, r_{1}=1, \alpha_{1}=\frac{4 \pi}{3}, r_{2}=1, \alpha_{2}=\frac{4 \pi}{3} .
$$

If $c_{1}=0$, then by (3.13), $c_{0}=c_{2}=1$, which leads, by (2.13), to

$$
r_{1}=0, r_{0}=1, \alpha_{0}=0, r_{2}=1, \alpha_{2}=2 \pi
$$

If $c_{2}=0$, then by (3.13), $c_{0}=c_{1}=1$, which leads, by (2.13), to

$$
r_{2}=0, r_{0}=1, \alpha_{0}=\frac{2 \pi}{3}, r_{1}=1, \alpha_{1}=\frac{2 \pi}{3} .
$$

In all the cases (3.15), (3.16) and (3.17), $r_{k} \mathrm{e}^{\mathrm{i} \alpha_{k}} \in T_{k} \cup\{0\}$ for $k \in\{0,1,2\}$. Then (2.11) implies, by Lemma 3.1, that

$$
f=\sum_{k=0}^{2} r_{k} \mathrm{e}^{\mathrm{i} \alpha_{k}} \cdot \chi_{T_{k}} \text { a.e. on } \mathbb{T} \text {. }
$$

In particular, (3.18) together with (3.15), (2.25) and (3.5) yields

$$
f=0 \cdot \chi_{T_{0}}+e_{2} \cdot \chi_{T_{1}}+e_{2} \cdot \chi_{T_{2}}=e_{2} \cdot \chi_{T_{1} \cup T_{2}}=S_{2} \circ f_{0} \circ S_{2}^{-1} \text { a.e. on } \mathbb{T} \text {. }
$$

From (3.18), (3.16), (2.25) and (3.5) we see that

$$
f=0 \cdot \chi_{T_{1}}+e_{0} \cdot \chi_{T_{0}}+e_{0} \cdot \chi_{T_{2}}=e_{0} \cdot \chi_{T_{0} \cup T_{2}}=S_{0} \circ f_{0} \circ S_{0}^{-1} \text { a.e. on } \mathbb{T} \text {. }
$$


Finally, (3.18), (3.17), (2.25) and (3.5) lead to

$$
f=0 \cdot \chi_{T_{2}}+e_{1} \cdot \chi_{T_{0}}+e_{1} \cdot \chi_{T_{1}}=e_{1} \cdot \chi_{T_{0} \cup T_{1}}=S_{1} \circ f_{0} \circ S_{1}^{-1} \text { a.e. on } \mathbb{T} \text {. }
$$

Therefore all these equalities yield the second part of the conjunction in (3.10), provided $z \neq 0$, and the first part of the alternative in (3.11), provided $z=0$. Assuming now that $r=|z|>0$, we conclude from (2.19) that

$$
Q_{r}(\theta)>Q_{r}(0), \quad \theta \in \mathbb{R} \backslash\{2 n \pi: n \in \mathbb{Z}\}
$$

For each $k \in\{0,1,2\}$ we conclude from (3.14) that if $c_{k}=0$, then $p_{k}=Q_{r}(0)$. Therefore, from (3.19), (2.21) and (3.5) it follows that:

If $c_{0}=0$, then $\theta_{r}=4 \pi / 3$, and so $z=S_{2}(|z|)$.

If $c_{1}=0$, then $\theta_{r}=0$, and so $z=S_{0}(|z|)$.

If $c_{2}=0$, then $\theta_{r}=2 \pi / 3$, and so $z=S_{1}(|z|)$.

These equalities yield the first part of the conjunction in (3.10), provided $z \neq 0$.

It remains to examine the case where $0<c \leq 1$. Then all $c_{0}, c_{1}, c_{2}$ are positive. Analyzing the part of the proof of Theorem 2.1 from (2.15) until (2.17) we get the following reasoning: From (3.13) we deduce that for a given $\alpha \in I_{0}$,

$$
2=c_{0}+c_{1}+c_{2} \leq 1+2 \cos \frac{\pi}{3} \cos \left(\frac{\pi}{3}-\alpha\right),
$$

whence $\alpha=\pi / 3$. Then $c_{0} \leq 1, c_{1} \leq 1 / 2$ and $c_{2} \leq 1 / 2$. Applying (3.13) once more we get $c_{0}=1, c_{1}=1 / 2$ and $c_{2}=1 / 2$. Since

$$
c_{k}=r_{k} \cos \left(\alpha_{k}-\frac{\pi}{3}\right), \quad 0 \leq r_{k} \leq 1 \quad \text { and } \quad \alpha_{k} \in I_{k}, \quad k \in\{0,1,2\}
$$

we see that

$$
r_{0}=1, \alpha_{0}=\frac{\pi}{3}, r_{1}=1, \alpha_{1}=\frac{2 \pi}{3}, r_{2}=1, \alpha_{2}=2 \pi
$$

In the similar manner we show that:

If $\alpha \in I_{1}$, then $\alpha=\pi, c_{0}=1 / 2, c_{1}=1, c_{2}=1 / 2$, and consequently

$$
r_{0}=1, \alpha_{0}=\frac{2 \pi}{3}, r_{1}=1, \alpha_{1}=\pi, r_{2}=1, \alpha_{2}=\frac{4 \pi}{3}
$$

If $\alpha \in I_{2}$, then $\alpha=5 \pi / 3, c_{0}=1 / 2, c_{1}=1 / 2, c_{2}=1$, and consequently

$$
r_{0}=1, \alpha_{0}=0, r_{1}=1, \alpha_{1}=\frac{4 \pi}{3}, r_{2}=1, \alpha_{2}=\frac{5 \pi}{3}
$$

In all the cases (3.20), (3.21) and (3.22), $r_{k} \mathrm{e}^{\mathrm{i} \alpha_{k}} \in T_{k}$ for $k \in\{0,1,2\}$. Then (2.11) implies, by Lemma 3.1, the equality (3.18). In particular, (3.18) together with (3.20), 
(3.12) and (3.5) yields

$$
f=\mathrm{e}^{\pi \mathrm{i} / 3} \cdot \chi_{T_{0}}+e_{1} \cdot \chi_{T_{1}}+e_{0} \cdot \chi_{T_{2}}=f_{0}^{*}=S_{0} \circ f_{0}^{*} \circ S_{0}^{-1} \text { a.e. on } \mathbb{T} \text {. }
$$

From (3.18), (3.21), (3.12) and (3.5) we see that

$$
f=e_{1} \cdot \chi_{T_{0}}+\mathrm{e}^{\pi \mathrm{i}} \cdot \chi_{T_{1}}+e_{2} \cdot \chi_{T_{2}}=S_{1} \circ f_{0}^{*} \circ S_{1}^{-1} \text { a.e. on } \mathbb{T} \text {. }
$$

Finally, (3.18), (3.22), (3.12) and (3.5) lead to

$$
f=e_{0} \cdot \chi_{T_{0}}+e_{2} \cdot \chi_{T_{1}}+\mathrm{e}^{5 \pi \mathrm{i} / 3} \cdot \chi_{T_{2}}=S_{2} \circ f_{0}^{*} \circ S_{2}^{-1} \text { a.e. on } \mathbb{T} \text {. }
$$

Therefore all these equalities yield the second part of the alternative in (3.11). Moreover, as shown above, $c_{i}=c_{j}=1 / 2$ for some $i, j \in\{0,1,2\}$ such that $i \neq j$. Then by the condition (3.14) it follows that $p_{i}=p_{j}=Q_{r}(0)$. However, from (2.21) and (3.19) we see that this is impossible provided $|z|=r>0$. Therefore, $z=0$, which completes the proof.

Corollary 3.4 For any function $F \in \operatorname{Har}(\mathbb{D})$ and $z \in \mathbb{D}$, if $F(\mathbb{D}) \subset \mathbb{D}$ and the condition (2.28) holds for every $k \in\{0,1,2\}$, then

$$
|F(z)|=\frac{4}{3}-\frac{2}{\pi} \arctan \left(\frac{\sqrt{3}}{1+2|z|}\right)
$$

iff there exists $k \in\{0,1,2\}$ such that one of the two following conditions holds:

(i) $z \neq 0$ and

$$
z=S_{k}(|z|) \text { and } F(u)=S_{k} \circ F_{0} \circ S_{k}^{-1}(u), \quad u \in \mathbb{D} \text {, }
$$

where $F_{0}:=\mathrm{P}\left[f_{0}\right]$ with $f_{0}$ given by the formula (2.25);

(ii) $z=0$ and

$$
F(u)=S_{k} \circ F_{0} \circ S_{k}^{-1}(u) \text { or } F(u)=S_{k} \circ F_{0}^{*} \circ S_{k}^{-1}(u), \quad u \in \mathbb{D},
$$

where $F_{0}^{*}:=\mathrm{P}\left[f_{0}^{*}\right]$ with $f_{0}^{*}$ given by the formula (3.12).

Proof From the definitions of the classes $\mathcal{H}$ and $\mathcal{F}$ it follows that $\mathcal{H}=\mathrm{P}[\mathcal{F}]$; cf. [3, Cor. 1 and Cor. 2, Sect. 1.2]. Thus the corollary follows directly from Theorem 3.3, the property (3.8) as well as the equalities $F_{0}=\mathrm{P}\left[f_{0}\right]$ and $F_{0}^{*}=\mathrm{P}\left[f_{0}^{*}\right]$.

Remark 3.5 The formula (3.1) gives an explicite form of the function $F_{0}$. From (2.30) we have

$$
\mathrm{P}\left[\chi_{T_{1}}\right]=\mathrm{P}\left[1-\chi_{T_{0} \cup T_{2}}\right]=1-\mathrm{P}\left[\chi_{T_{0} \cup T_{2}}\right]=1-F_{0} .
$$

Hence and by (3.5) we see that

$$
\begin{aligned}
& \mathrm{P}\left[\chi_{T_{0}}\right]=\mathrm{P}\left[\chi_{T_{1}} \circ S_{2}^{-1}\right]=\mathrm{P}\left[\chi_{T_{1}}\right] \circ S_{2}^{-1}=1-F_{0} \circ S_{1}, \\
& \mathrm{P}\left[\chi_{T_{2}}\right]=\mathrm{P}\left[\chi_{T_{1}} \circ S_{1}^{-1}\right]=\mathrm{P}\left[\chi_{T_{1}}\right] \circ S_{1}^{-1}=1-F_{0} \circ S_{2} .
\end{aligned}
$$


Using now the formula (3.12) we deduce that

$$
\begin{aligned}
F_{0}^{*} & =\mathrm{P}\left[f_{0}^{*}\right]=\mathrm{e}^{\pi \mathrm{i} / 3} \cdot \mathrm{P}\left[\chi_{T_{0}}\right]+e_{1} \cdot \mathrm{P}\left[\chi_{T_{1}}\right]+e_{0} \cdot \mathrm{P}\left[\chi_{T_{2}}\right] \\
& =\mathrm{e}^{\pi \mathrm{i} / 3}\left(\left(1-F_{0} \circ S_{1}\right)+\mathrm{e}^{\pi \mathrm{i} / 3}\left(1-F_{0}\right)+\mathrm{e}^{-\pi \mathrm{i} / 3}\left(1-F_{0} \circ S_{2}\right)\right) \\
& \left.=\mathrm{e}^{\pi \mathrm{i} / 3}\left(2-F_{0} \circ S_{1}-\mathrm{e}^{\pi \mathrm{i} / 3} F_{0}-\mathrm{e}^{-\pi \mathrm{i} / 3} F_{0} \circ S_{2}\right)\right) .
\end{aligned}
$$

Thus we obtain the following explicite form of the function $F_{0}^{*}$,

$$
F_{0}^{*}(z)=\mathrm{e}^{\pi \mathrm{i} / 3}\left(2-F_{0}\left(\mathrm{e}^{2 \pi \mathrm{i} / 3} z\right)-\mathrm{e}^{\pi \mathrm{i} / 3} F_{0}(z)-\mathrm{e}^{-\pi \mathrm{i} / 3} F_{0}\left(\mathrm{e}^{4 \pi \mathrm{i} / 3} z\right)\right), \quad z \in \mathbb{D},
$$

which leads to the more symmetric one

$$
\mathrm{e}^{-\pi \mathrm{i} / 3} F_{0}^{*}\left(\mathrm{e}^{\pi \mathrm{i} / 3} z\right)=2-F_{0}(-z)-\mathrm{e}^{\pi \mathrm{i} / 3} F_{0}\left(\mathrm{e}^{\pi \mathrm{i} / 3} z\right)-\mathrm{e}^{-\pi \mathrm{i} / 3} F_{0}\left(\mathrm{e}^{-\pi \mathrm{i} / 3} z\right), \quad z \in \mathbb{D} .
$$

Open Access This article is distributed under the terms of the Creative Commons Attribution License which permits any use, distribution, and reproduction in any medium, provided the original author(s) and the source are credited.

\section{References}

1. Ahlfors, L.V.: Lectures on Quasiconformal Mappings. D. Van Nostrand, Princeton, Toronto, New York, London (1966)

2. Choquet, G.: Sur les homéomorphies harmoniques d'un disque $D$ sur $D$. Complex Var. 24, 47-48 (1993)

3. Duren, P.: Theory of $H^{p}$-spaces. Dover Publications Inc., Mineola, New York (2000)

4. Duren, P.: Harmonic Mappings in the Plane, Cambridge Tracts in Mathematics. Cambridge University Press, Cambridge (2004)

5. Heinz, E.: On one-to-one harmonic mappings. Pacif. J. Math. 9, 101-105 (1959)

6. Hengartner, W., Schober, G.: Harmonic mappings with given dilatation. J. Lond. Math. Soc. 33(2), 473-483 (1986)

7. Heyman, W.K., Kennedy, P.B.: Subharmonic Functions, vol. I. Academic Press, London (1976)

8. Partyka, D., Sakan, K.: Three variants of Schwarz's lemma for harmonic mappings, Bull. Soc. Sci. Lett. Łódź 52, 27-34 (2002) (Série: Recherches sur les déformations 36) 